\title{
Osteogenic Potential of Oxycellulose - A Molecular-Biological and Histological Study in Rats
}

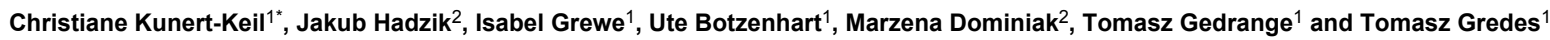 \\ ${ }^{1}$ Department of Orthodontics, Carl Gustav Carus Campus, Technische Universität Dresden, Germany \\ ${ }^{2}$ Department of Dental Surgery Wroclaw Medical University, Wroclaw, Poland
}

*Corresponding author: Christiane Kunert-Keil, Fetscherstr. 74, Haus 28, D-01307 Dresden, Germany, Tel: 0049-351-4582718; Fax: 0049-351-4585318; E-mail: christiane.kunert-keil@uniklinikum-dresden.de

Rec date: Dec 11, 2014, Acc date: Jan 05, 2015, Pub date: Jan 07, 2015

Copyright: (c) 2015 Kunert-Keil C, et al. This is an open-access article distributed under the terms of the Creative Commons Attribution License, which permits unrestricted use, distribution, and reproduction in any medium, provided the original author and source are credited.

\begin{abstract}
Oxidized cellulose is a polysaccharide substance formed by the oxidation of cellulose. Depending on the amount used, oxycellulose is completely absorbable and can be used as hemostatic agents in general and dental surgery for hemostatic purposes. Each procedure on the bone also carries the risk of bleeding, but still not much is known about the bone tissue reaction on this material. Due to this fact, the aim of this study was to evaluate the tissue reaction and osteogenic potential of the natural oxycellulose material RESORBA-CELL $®$ standard by its implantation into rat cranial bone defects. The materials were evaluated using an established rat cranial defect model in 22 animals. One bone defect with a diameter of $5 \mathrm{~mm}$ was created per animal. The defects were filled with oxycellulose and left to heal for 4 weeks. Twelve samples ( $n=6$ oxycellulose; $n=6$ untreated control lesions) were processed for histological evaluation. The remaining 10 samples were processed for mRNA expression analysis of genes coding for growth factors and osteogenic differentiation using quantitative RT-PCR. In oxycellulose treated bone lesions significant reduced mRNA levels of Runx2, Bglap and COL1A1 were found. The expression reached $74 \%, 73 \%$ and $47 \%$ of the mRNA quantity of the untreated bone, respectively. In the histological sections, the bone defects were completely filled with connective tissue / bone marrow with embedded foam cells after four weeks. In contrast to the controls, the tested material do not induced early bone healing. The residual bone seems to be rather absorbed. In conclusion, the study has shown that the RESORBA-CELL $®$ oxidized cellulose has no osteogenic potential, but it still can be considered as a useful wound dressing in the dental surgery.
\end{abstract}

Keywords: Oxycellulose; Osteogenic potential, Rat cranial defect; Histology; RT-PCR

\section{Introduction}

Bone is the most commonly transplanted tissue after blood. Worldwide, estimated 2.2 million grafting procedures are performed annually to repair bone defects in orthopaedics, neurosurgery, and dentistry [1]. The increasing number of grafting procedures and the disadvantages of current autograft and allograft treatments (e.g. limited graft quantity, risk of disease transmission) drive the quest for alternative methods to treat bone defects. The use of synthetic bioactive bone substitute materials is of increasing importance in modern dentistry as alternatives to autogenous bone grafts. Bone graft materials are generally evaluated based on their osteogenic, osteoinductive, or osteoconductive potential.

Osteoinduction is a basic biological mechanism that occurs regularly in any type of bone healing process. Osteoinduction process starts immediately after the bone injury. This process induces a cascade of events including primitive, undifferentiated and pluripotent cells to develop into the bone-forming cells - pre-osteoblasts [2,3]. In other words the molecular mechanism of this phenomenon is to use the immature cells, and to stimulate them, so the osteogenesis process can occur. It is a dynamic process that is responsible for healing and the continuous remodeling of bone tissue. There are several cell-type specific markers that can be identified in order to characterize ongoing cellular differentiation, and bone remodeling process. Some of these, like Runx2, alkaline phosphatase (Alpl), osteocalcin (bone gammacarboxyglutamate protein, Bglap), Acp5 (acid phosphatase type 5) and Phex, belong to the key phenotyping proteins expressed in the osteogenic cell lineage [3-6].

Runt-related transcription factor 2 (Runx2) is also called CBFA1 (core binding factor 1). It is essential for the osteoblast differentiation. This factor acts throughout the induction, proliferation, and maturation of osteoblasts and regulates expression of many osteoblast genes $[7,8]$. Runx2 is an essential factor in the development of the skeletal system; studies showed that lack of the Runx2 results in an immediate postnatal death of Runx2-deficient mice due to a complete lack of mineralized bone [9-11]. Mutations in the Runx2 gene result in specific diseases, haploinsufficiency causes Cleidocranial dysplasia (CCD) a condition characterized by hypoplastic clavicles and a number of other skeletal abnormalities [11]. Runx2 is also a specific regulator of osteoblast gene expression like osteocalcin, Alpl and collagen type I [12]. Osteocalcin is also known as bone gammacarboxyglutamic acid-containing protein (BGLAP), a non-collagenous protein found in bone and dentin. Osteocalcin is secreted solely by maturing osteoblasts and is implicated in bone mineralization and calcium ion homeostasis in human body [12]. Collagen type I is a protein which is the major component of many extracellular matrices. It is synthesized by a small number of discrete cell types, including fibroblasts, osteoblasts and odontoblasts. The COL1A1 gene encodes this protein in humans. Mutations in the COL1A1 gene are associated with severe systemic disturbances such as Ehlers-Danlos syndrome or Osteogenesis imperfecta [13]. 
Page 2 of 5

To date, predominantly histological staining and immunohistochemical analyses were used to study the behaviour of bone substitute materials on bone graft healing. Only a few numbers of studies present the molecular mechanisms of bone formations associated with bone substitutes. Recently it was shown, that hydroxyapatite (HA) and $\beta$-tricalcium phosphate $(\beta$-TCP) up-regulate the expression of Runx2, Alpl, Acp5 and osteocalcin in biopsies from human jaws [14] and rat skulls [4,6]. When using flax composites for the treatment of bone defects significantly higher expression of IGF1 and decreased mRNA levels of Bglap and MMP8 were found compared to untreated controls [5]. Nevertheless, flax composites are able to stimulate bone regeneration. Main component of flax fibres is the natural polymer cellulose. It is well known that cellulose is hydrophilic, insoluble in water as well as most organic solvents and not biodegradable.

Oxidized cellulose is a polysaccharide substance formed by the oxidation of cellulose. It is obtained by means of oxidation of highly pure cellulose. It can be prepared in different physical forms, such as powder, textile, paper or fiber form [15]. Depending on the amount used, oxycellulose is absorbable [16,17]. Oxidized cellulose can be used as hemostatic agents in general and dental surgery for hemostatic purposes [15-18]. Each procedure on the bone also carries the risk of bleeding, but still not much is known about the bone tissue reaction on this material. Due to this fact, the aim of this study was to evaluate the tissue reaction and osteogenic potential of the natural oxycellulose material RESO-CELL by its implantation into rat cranial bone defects.

\section{Material and Methods}

\section{Test material}

Sterile absorbable RESORBA ${ }^{\circ}$ CELL standard $(1 \times 0.5 \mathrm{~cm}$, Resorba Wundversorgung, Nürnberg, Germany) gauze strips are produced from natural cotton cellulose by controlled oxidation. The product is white and does not fray when cut (Resorba Medical GmbH 2013).

\section{Experimental design and surgical procedure}

Twenty-two adult Lewis 1A rats (2-month old, body weight between $250 \mathrm{~g}$ and $350 \mathrm{~g}$ and of both sexes) were used for this study. All surgical and experimental procedures were approved by the Animal Welfare Committee on the State Government (LALLF MV/TSD/7221.3-1.1-033/11). For surgery, each rat was anesthetized with intraperitoneal injection of Ketamine (10\%; CEVA Tiergesundheit, Düsseldorf, Germany) and Rompun (2\%; Bayer HealthCare, Leverkusen, Germany) with a ratio of 3:2 and at an approximate dosage of $0.1 \mathrm{ml} / 100 \mathrm{~g}$ body weight. A midline skin incision was performed on the skull. Bone defects with a diameter of 5 $\mathrm{mm}$ size (using a pre-designed template) were created in each parietal region of the cranium with a trephine under constant irrigation as described previously (Figure 1A) $[4-6,19]$. Bone defects $(n=16)$ were covered with oxycellulose pieces (RESORBA ${ }^{\circ}$ Cell standard; Figure 1B and 1C) and the scalp was closed with wound clips. Six untreated animals served as controls.
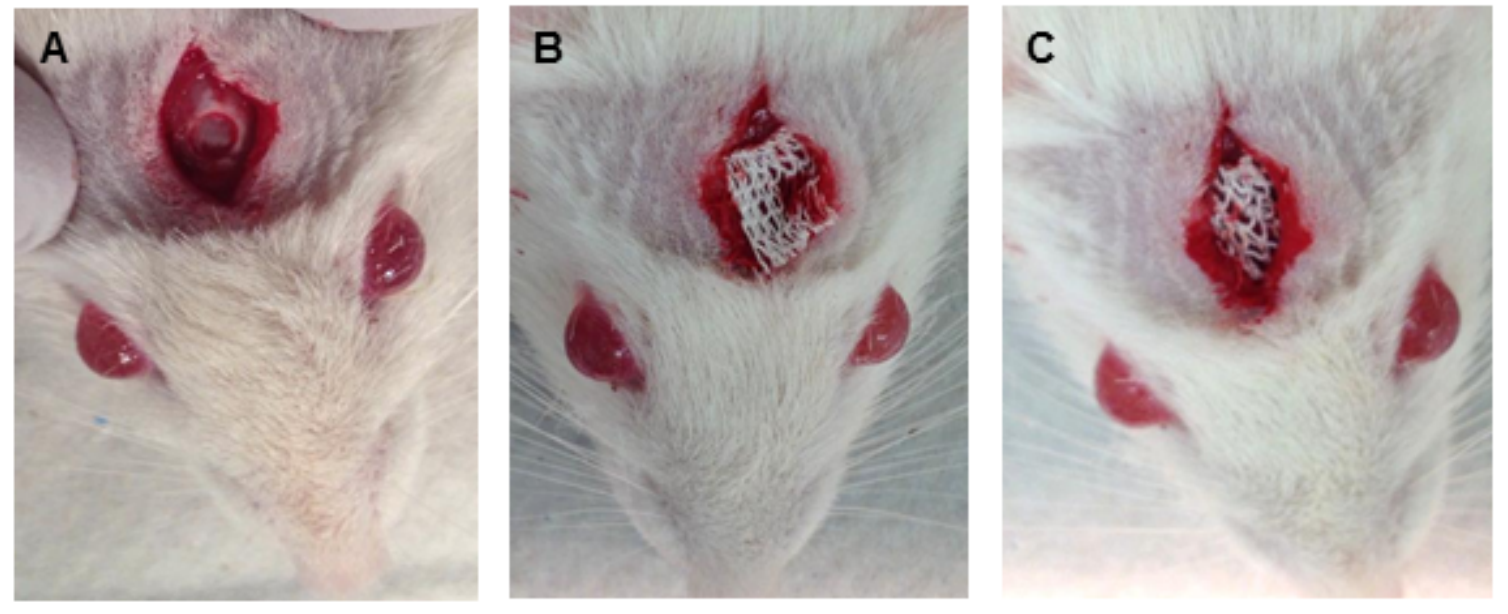

Figure 1: Rat cranium after preparation of the bone defect $(A)$ and placement of the oxycellulose into the bone $\operatorname{defect}(B+C)$.

The animals were sacrificed at four weeks. The skulls were harvested and processed for gene expression analysis and histological examination. For molecular-biological examination, dissected skulls (area of the treated lesion and untreated initial bone) were shockfrozen in liquid nitrogen and stored at $-80^{\circ} \mathrm{C}$. For histological examination, skulls were placed in $4 \%$ PBS-buffered formalin for one week at room temperature and then decalcified for one week in ethylenediaminetetraacetic acid (EDTA). After decalcification the bone cores were rinsed with running water for $24 \mathrm{~h}$; routinely dehydrated in a series of increasing concentrations of ethanol $(50 \%$, $70 \%, 80 \%, 90 \%, 96 \%$, and $100 \%$ ) placed in xylol for $12 \mathrm{~h}$ and then embedded in paraffin.

\section{RNA isolation, reverse transcription and TaqMan RT-PCR}

Homogenization of bone samples was performed in QIAzol lysis reagent (RNeasy Lipid Tissue Mini Kit, Qiagen, Hilden, Germany) using a mortar and pestle. The isolation of the total RNA was done using guanidinium-isothiocyanate (RNeasy Lipid Tissue Mini Kit, Qiagen, Hilden, Germany). An amount of $200 \mathrm{ng}$ total RNA was reverse transcribed (TaqMan-reverse transcription kit, PE Applied Biosystems, Weiterstadt, Germany).

To quantify the expression of different rat genes, gene-specific TaqMan PCR primers and probes were purchased from PE Applied 
Biosystems and quantitative real-time PCRs using a Toptical cycler (Biometra, Germany) were performed as described previously [4-6].

\section{Histological processing}

Serial longitudinal sections of about $5 \mu \mathrm{m}$ were stained with hematoxylin/eosin (H.E.) for recognizing various tissue types and Masson Goldner trichrome for differentiation between collagen, muscle and bone tissue.

A blind histological evaluation of all sections stained was undertaken by two independent investigators. The slices were observed and photographed under a Nikon light microscope (Eclipse E600) equipped with a calibrated digital camera (DXM1200, Nikon, Tokyo, Japan). Histomorphometric measurements were performed on images at a magnification of $10 \times$ using image analysis software (Image J, NIH, v. 1.61).

\section{Statistical analysis}

All statistical analyses were performed using the SigmaStat 3.5 Software (Systat Software, Inc.,1735, Technology Drive, San Jose, CA 95110, USA). Statistical analyses were made using the Mann-Whitney rank sum test. Data are given as means \pm S.D. $\mathrm{P}<0.05$ was considered statistically significant.

\section{Results}

\section{Real-time RT-PCR}

All the animals have recovered without problems from the surgery. No complications such as allergic reactions, abscesses or infections were observed throughout the study periods.

For weeks after oxycellulose insertion the animals were sacrificed, rat skulls prepared and dissected in two parts: 1) treated lesion and 2) untreated initial bone tissue. Both bone samples of each rat were used for molecular-biological analyses and the intra-individual gene expression of bone specific genes in both skull parts was compared to each other (Table 1). In oxycellulose treated bone lesions significant reduced mRNA levels of Runx2, Bglap and COL1A1 were found. The expression reached $74 \%, 73 \%$ and $47 \%$ of the mRNA quantity of the untreated bone, respectively. All other tested genes do not show any difference compared to controls (Table 1).

\begin{tabular}{|l|l|l|l|}
\hline \multirow{2}{*}{ Gene } & \multicolumn{2}{|c|}{ Bone tissue of } & P value \\
\cline { 2 - 4 } & Treated lesion & Untreated area & n.s. \\
\hline Acp5 & $0.12 \pm 0.024$ & $0.116 \pm 0.015$ & n.s. \\
\hline Alpl & $0.096 \pm 0.017$ & $0.084 \pm 0.009$ & $P=0.002$ \\
\hline Bglap & $0.87 \pm 0.13$ & $1.85 \pm 0.25$ & $P=0.041$ \\
\hline Col1a1 & $3.04 \pm 0.56$ & $4.15 \pm 0.42$ & n.s. \\
\hline IGF1 & $0.46 \pm 0.09$ & $0.39 \pm 0.05$ & n.s. \\
\hline IGF2 & $1.17 \pm 0.18$ & $1.04 \pm 0.17$ & n.s. \\
\hline Phex & $0.073 \pm 0.022$ & $0.031 \pm 0.002$ & $P=0.015$ \\
\hline Runx2 & $0.002 \pm 0.0002$ & $0.0027 \pm 0.0003$ & n.s. \\
\hline VEGFA & $0.093 \pm 0.015$ & $0.095 \pm 0.015$ & \\
\hline
\end{tabular}

Table 1: Gene specific transcription levels in rat bone tissue samples. The mRNA levels are given in relation to those of $18 \mathrm{~S}$ rRNA. Means \pm S.E.M. are given in all cases for $\mathrm{n}=10$ samples. $\mathrm{P}$ values indicate statistical significant differences between bone tissue of treated lesions and untreated initial bone tissue, Mann Whitney U rank sum test. n.s.=not significant.

\section{Histology}

In the histological sections, there were no signs of a cellular inflammatory infiltrate and reaction against foreign bodies. The untreated defects were completely filled with connective tissue after four weeks. Furthermore, the onset of bone regeneration was found. The amount of regenerated bone was approximately $47.8 \pm 1.3 \%$. Likewise untreated defects, in oxycellulose treated defects cell-rich areas of connective tissue/bone marrow were observed. Embedded in the connective tissue areas with foam cells (transformed macrophages) were found (Figure 2). In contrast to the controls, the tested material do not induced early bone healing. The residual bone seems to be rather absorbed (Figure 2).

\section{Discussion}

Oxidized cellulose materials are usually placed in the surgical site to achieve hemostasis $[16-18,20]$. They can be used in surgical and minimally invasive procedures to support hemostasis in capillary, venous, and minor diffuse arterial bleeding, in which conventional hemostatic measures such as sutures or ligatures are ineffective or unfeasible $[16-18,20]$. In addition to topical hemostasis, the bacteriostatic and antiseptic properties of oxycellulose products can prevents the secondary infection by inhibiting the growth and spread of gram-positive and gram-negative microorganisms and aerobic and anaerobic bacteria $[17,21,22]$. Manipulations and intervention in the bone can also bleed profusely. Normally such bleeding bone was treated with bone wax [17]. In our study surgically prepared bleeding bone defects were covered with oxycellulose pieces and the tissue 
reaction to the material was molecular-biological and histological examined.

A

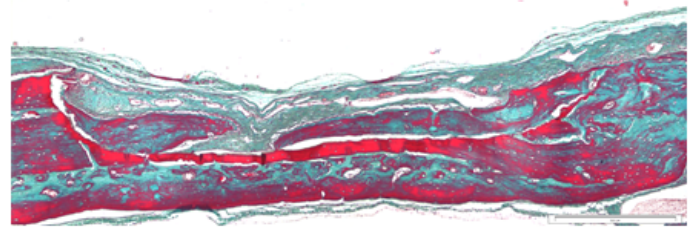

B

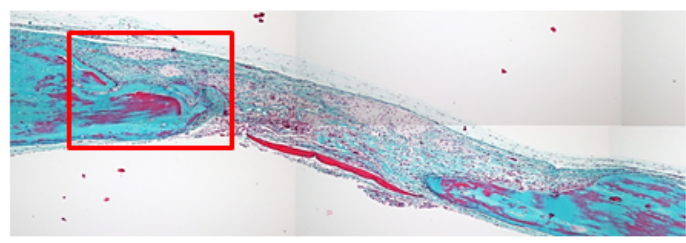

C

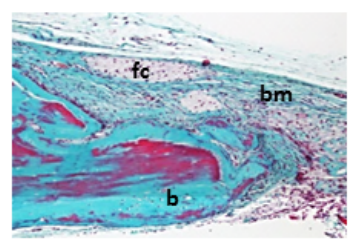

Figure 2: Masson Goldner trichrome staining of a calvarial crosssection embedded in paraffin after 4 weeks of healing. (A) untreated control lesion, (B) oxycellulose treated bone lesion, magnification $\mathrm{x} 20$; (C) higher magnification of the highlighted area (red frame), magnification $\mathrm{x} 100$. $\mathrm{b}=$ bone; bm=bone marrow / connective tissue; $\mathrm{fc}=$ foam cells.

Recent studies indicate quick resolution and complete resorption of the oxidized cellulose material during the second week after implantation [23]. Otherwise some reports suggest that oxycellulose dressings used in general surgery can swell and increase its volume leading to mechanical compression on vital anatomical structures and radiographic artifacts mimicking abscesses and tumors in CT and ultrasonography $[24,25]$. In our study, four weeks after implantation of the oxycellulose, no intact material could be detected. The oxidized cellulose was completely resorbed from macrophages. Transformed macrophages (foam cells) were found embedded in the connective tissue, which fill the bone defect. Furthermore, the tested material in our study did not show any osteogenic potential. Moreover it partially inhibited early bone healing as compared to control. The results indicate that the mRNA amount of Runx2 was statistical significantly lower after treatment with oxycellulose. The mRNA levels of osteocalcin (Bglap) and Col1a1 were also statistically significant decreased in treated bone lesions compared to untreated initial bone tissue. The decrease of the Runx 2 shows that tested material inhibits the maturation of the osteoblasts. Runx2 as a specific regulator of osteoblast gene expression may have also influenced the expression level of osteocalcin and collagen type I. The results obtained by molecular biology were confirmed by the histological preparations. Oxycellulose treated bone defects showed no bone regeneration, and resorption of remaining bone. Our findings are consistent with a number of previous studies [17,26-29]. Armstrong et al. examined bone defect treated with the oxidized regenerated cellulose. Healing of the bone defects was evaluated by micro-computed tomography (micro-CT) and histological research. His study showed that tested material inhibited early bone healing [26]. Ibarrola et al. studied bone healing in a rat tibial bone defects using light microscopy. In this study the oxidized cellulose caused an intense inflammatory response and bone repair was reduced [27]. A subperiosteal implantation of oxidized cellulose in five patients demonstrated no radiographic evidence of bone formation after 12 months [28]. In contrast, bone regeneration in surgical defects in the iliac crest of dogs was found after application of oxidized cellulose [30].

In conclusion, the study has shown that the RESORBA-CELL oxidized cellulose has no osteogenic potential; moreover it partially inhibits an early healing of the osseous defects in rats. Although the substance does slightly negatively affect the healing of the bone defect it still can be considered as a useful wound dressing in the dental surgery because of its hemostatic, bacteriostatic and antiseptic properties.

\section{Acknowledgement}

We would like to thank Michaela Krause for the excellent technical assistance and Resorba Wundversorgung GmbH (Nürnberg, Germany) for providing the research materials.

\section{References}

1. Giannoudis PV, Dinopoulos H, Tsiridis E (2005) Bone substitutes: an update. Injury 36 Suppl 3: S20-27.

2. Albrektsson T, Johansson C (2001) Osteoinduction, osteoconduction and osseointegration. Eur Spine J 10 Suppl 2: S96-101.

3. Kunert-Keil C, Gredes T, Gedrange T (2011) Biomaterials Applicable for Alveolar Sockets Preservation: In Vivo and In Vitro Studies. Implant Dentistry - The Most Promising Discipline of Dentistry, InTech.

4. Gredes T, Kracht T, Gedrange T, Kunert-Keil C (2012) Influence of BONITmatrix $\left({ }^{\oplus}\right)$ and OSSA NOVA on the expression of bone specific genes. Ann Anat 194: 524-528.

5. Gredes T, Wróbel-Kwiatkowska M, Dominiak M, Gedrange T, KunertKeil C (2012) Osteogenic capacity of transgenic flax scaffolds. Biomed Tech (Berl) 57: 53-58.

6. Kunert-Keil C, Scholz F, Gedrange T, Gredes T (2013) Comparative study of biphasic calcium phosphate with beta-tricalcium phosphate in rat cranial defects-A molecular-biological and histological study. Ann Anat.

7. Jensen ED, Gopalakrishnan R, Westendorf JJ (2010) Regulation of gene expression in osteoblasts. Biofactors 36: 25-32.

8. Pratap J, Galindo M, Zaidi SK, Vradii D, Bhat BM, et al. (2003) Cell growth regulatory role of Runx2 during proliferative expansion of preosteoblasts. Cancer Res 63: 5357-5362.

9. Komori $\mathrm{T}$ (2003) Requisite roles of Runx2 and $\mathrm{Cbfb}$ in skeletal development. J Bone Miner Metab 21: 193-197.

10. Komori T, Yagi H, Nomura S, Yamaguchi A, Sasaki K, et al. (1997) Targeted disruption of Cbfal results in a complete lack of bone formation owing to maturational arrest of osteoblasts. Cell 89: 755-764.

11. Otto F, Kanegane H, Mundlos S (2002) Mutations in the RUNX2 gene in patients with cleidocranial dysplasia. Hum Mutat 19: 209-216.

12. Ducy P (2000) Cbfa1: a molecular switch in osteoblast biology. Dev Dyn 219: 461-471.

13. Rossert J, Terraz C, Dupont S (2000) Regulation of type I collagen genes expression. Nephrol Dial Transplant 15 Suppl 6: 66-68.

14. Gotz W, Gerber T, Michel B, Lossdorfer S, Henkel KO, et al. (2008) Immunohistochemical characterization of nanocrystalline 
Citation: Kunert-Keil C, Hadzik J, Grewe I, Botzenhart U, Dominiak M (2015) Osteogenic Potential of Oxycellulose - A Molecular-Biological and Histological Study in Rats. J Cytol Histol 6: 304. doi:10.4172/2157-7099.1000304

Page 5 of 5

hydroxyapatite silica gel (NanoBone(r)) osteogenesis: a study on biopsies from human jaws. Clin. Oral. Implants Res 19: 1016-1026.

15. Bajerova M, Krejcova K, Rabiskova M, Gajdziok J, Masteikova R (2009) Oxycellulose: significant characteristics in relation to its pharmaceutical and medical application. Adv Polym Technol 28: 199-208.

16. Bechstein WO, Strey C (2007) [Local and systemic hemostasis in surgery]. Chirurg 78: 95-96, 98-100.

17. Schonauer C, Tessitore E, Barbagallo G, Albanese V, Moraci A (2004) The use of local agents: bone wax, gelatin, collagen, oxidized cellulose. Eur Spine J 13 Suppl 1: S89-96.

18. Kollár P, Suchý P, Muselík J, Bajerová M, Havelka P, et al. (2008) [Hemostatic effects of oxidized cellulose]. Ceska Slov Farm 57: 11-16.

19. Kunert-Keil C, Gredrange T, Mai R, Spassov A, Lucke S et al. (2009) Morphological evaluation of bone defect regeneration after treatment with two different forms of bone substitution materials on the basis of BONITmatrix. J Physiol Pharmacol 60 Suppl 8: 57-60.

20. Oto A, Remer EM, O'Malley CM, Tkach JA, Gill IS (1999) MR characteristics of oxidized cellulose (Surgicel). AJR Am J Roentgenol 172: 1481-1484.

21. Scher KS, Coil JA Jr (1982) Effects of oxidized cellulose and microfibrillar collagen on infection. Surgery 91: 301-304.

22. Spangler D, Rothenburger S, Nguyen K, Jampani H, Weiss S et al. (2003) In vitro antimicrobial activity of oxidized regenerated cellulose against antibiotic-resistant microorganisms. Surg Infect (Larchmt) 4: 255-262.
23. Witte B, Kroeber SM, Hillebrand H, Wolf M, Huertgen M (2013) Cotton-derived oxidized cellulose in minimally invasive thoracic surgery: a clinicopathological study. Innovations (Phila) 8: 296-301.

24. Ibrahim MF, Aps C, Young CP (2002) A foreign body reaction to Surgicel mimicking an abscess following cardiac surgery. Eur J Cardiothorac Surg 22: 489-490

25. Royds J, Kieran S, Timon C (2012) Oxidized cellulose (Surgicel) based reaction post thyroidectomy mimicking an abscess: A case report. Int J Surg Case Rep 3: 338-339.

26. Armstrong JK, Han B, Kuwahara K, Yang Z, Magyar CE, et al. (2010) The effect of three hemostatic agents on early bone healing in an animal model. BMC Surg 10: 37 .

27. Ibarrola JL, Bjorenson JE, Austin BP, Gerstein H (1985) Osseous reactions to three hemostatic agents. J Endod 11: 75-83.

28. Nappi JF, Lehman JA Jr (1980) The effects of Surgicel on bone formation. Cleft Palate J 17: 291-296.

29. Nooh N, Abdullah WA, Grawish Mel-A, Ramalingam S, Javed F, et al. (2014) The effects of surgicel and bone wax hemostatic agents on bone healing: An experimental study. Indian J Orthop 48: 319-325.

30. Finn MD, Schow SR, Schneiderman ED (1992) Osseous regeneration in the presence of four common hemostatic agents. J Oral Maxillofac Surg 50: 608-612. 